\title{
Benefits of Virtual Reality Used in Vestibular Rehabilitation in Adult and Elderly Individuals with Dizziness
}

\section{Salomão KS ${ }^{1}$, Sousa $\mathrm{MGC}^{2 *}$ and Machado $\mathrm{GC}^{3}$}

${ }^{1}$ Department of Life Sciences (DCV-I), State University of Bahia, Brazil

${ }^{2}$ Adjunct professor of the Phonoaudiology, State University of Bahia, Brazil

${ }^{3}$ Collaborator, Master in Interactive Processes of the Organs and Systems of the Institute of Health Sciences at UFBA, Brazil

*Corresponding author: Maria da Glória Canto de Sousa, Adjunct professor of the Phonoaudiology, State University of Bahia (UNEB), Salvador, BA, Brazil, Tel: +71988690903; Email: gcanto@uneb.br

\section{Research Article}

Volume 5 Issue 2

Received Date: July 22, 2020

Published Date: July 30, 2020

DOI: $10.23880 /$ ooaj-16000195

\section{Abstract}

Introduction: Aging brings about several changes in health in the elderly. Dizziness is one of the symptoms most commonly found in this age group, causing psychosocial disorders in the daily activities.

Objective: To assess the benefits of virtual reality by using the video game X-Box 360, in the vestibular rehabilitation of affected individuals of dizziness.

Materials and Methods: The research consists of an observational study of the quantitative type of exploratory and descriptive character. The participants were five individuals of both genders, with a mean age of 59.6 year, subject to fulfillment of Kinect games and Kinect Sport Adventure the X-Box 360, and evaluated by comparing the scores of Dizzines Handicap interventory DHI before and after intervention.

Results: $80 \%$ of participants are female, the gender and age did not influence the improvement of quality of life, $80 \%$ had reduction of DHI in the physical, functional and emotional domain and consequently improved quality of life.

Conclusion: The language intervention with patients with dizziness, through the video game X-Box 360, provided improvement in the quality of life of patients surveyed.

Keywords: Elderly; Dizziness; Rehab; Quality of life

\section{Introduction}

Aging causes several changes in the health of the elderly, causing functional organism changes [1]. As age advances, the vestibular system degenerates and dizziness appears [2]. According to studies, $85 \%$ of individuals over 65 years of age have dizziness [3]. Aging reduces the functionality of the vestibular system, increasing its prevalence [4]. With the increase in life expectancy, it is important to pay attention to the quality of life of the elderly, making them independent and active [5].
Dizziness is present in all age groups, but it is more often found in adults and especially in the elderly [3]. It is characterized by a sensation of altered body balance [6], and is classified according to the individual's perception, and can be rotational, when the person has the feeling that the environment around him is rotating or his own body rotates in environment, also being called vertigo; and non-rotational, when the individual does not have the perception of rotation, in this case, there is the disappearance of the spatial notion, sensation of fluctuation and body imbalance [2]. 


\section{Otolaryngology Open Access Journal}

Dizziness has negative impacts on quality of life and causes social, physical and psychological limitations [2]. It causes isolation of the individual in society, anxiety, depression and panic [7]. Individuals with dizziness report having a feeling of instability, imbalance, malaise, fluctuation, vomit, vertigo and neurovegetative symptoms [8], restricting the performance of daily activities in order to avoid injuries and / or embarrassments [9].

The assessment of quality of life through self-perception is essential to define the general consequences of vestibular disorders [10], thus allowing the measurement of the negative effects of dizziness in the performance of daily activities [11]. The Dizziness Handicap Inventory (DHI) questionnaire is the instrument commonly used to identify the impairment of dizziness in functional, physical and emotional aspects and to evaluate the benefits of vestibular rehabilitation (RV) on quality of life [12].

The RV program was created in the 40s by Cawthorne and Cooksey, the protocol consists of movement exercises and setting of the eyes, head, body and balance activities to stimulate the vestibular system, visual and proprioceptive [13]. This information is sent to the central nervous system, which will develop new neural arrangements, enabling neuroplasticity and, consequently, the creation of adaptive responses, through the mechanism of adaptation, habituation and substitution [14-16].

" $\mathrm{RV}$ is a physical exercise program associated with a set of measures and changes in habits that aim to accelerate vestibular compensation" [17], improving the individual's balance and restoring spatial orientation [18], through the repetition of these exercises without causing adverse effects [19]. Indicated and used in the therapy of individuals affected by altered balance caused by vestibular disorders, in which the main clinical characteristic is dizziness [11]. RV can be performed individually or in groups [4]. The success of the treatment or the recovery closer to normality will depend on the collaboration and commitment of the individual [16].

A strategy that has been used in RV is virtual reality, through visual stimulation and the simulation of individuals day to day moments [8]. It is worth mentioning that virtual reality also creates environments for the practice of sports activities [17], using the video game to make therapy playful. The use of electronic games in RV enables the production of proprioceptive, visual, vestibular and auditory stimuli, which provide the readjustment of the vestibulo-spinal and vestibulo-ocular reflex, important in the control of body balance, allowing the individual to interact with the game and others participants in real time8. Both conventional vestibular rehabilitation and RV with virtual reality have the same purpose of promoting stimulation, accelerating the compensation of the vestibular system and consequently the recovery of balance [20].

Despite the small amount of research using virtual reality in RV, there are satisfactory results [8]. Currently, few studies use X-Box 360 as an alternative resource for $\mathrm{RV}$, the video game is based on virtual reality and works as follows: the players' movements are captured by cameras in a space pre-determined by the video game, these movements are interpreted and transformed into commands allowing interaction between the game and the user [18].

Using technological tools, such as virtual reality, that help the rehabilitation process of individuals with dizziness, is very important, considering the psychosocial impacts on the adult and elderly population, which directly reflects on the quality of life of these subjects, in addition to disseminate and spread the use of video games in vestibular rehabilitation, justifying the relevance and importance of the present study, which aims to analyze the benefits of virtual reality, using the X-Box 360 video game, in the treatment of individuals affected by dizziness and identify the impact of dizziness on their quality of life.

\section{Materials and Methods}

The research in question was approved by the Research Ethics Committee of the State University of Bahia-UNEB, $\mathrm{n}^{\circ}$ CAAE 40339314.7.0000.0057, and consisted of an observational study of a quantitative type of exploratory and descriptive character. The field research was conducted at the Clinical School of Speech Teacher Jurandy Gomes de Aragon of Bahia State University (UNEB) in the city of Salvador, Bahia.

Five individuals participated in the research, four female and one male, aged between 58 and 61 years old, members of the Advanced Vestibular Rehabilitation Program developed at the Otoneurology Laboratory, from August to October 2015. Ten sessions, lasting approximately one hour each session. All subjects of the research had altered balance, such as dizziness, vertigo, headache, tinnitus, among others.

As an inclusion criterion, patients had to have performed the exercises of the conventional protocol (Cawthorne and Cooksey Protocol)13 for a period of approximately one year before entering the research and as an exclusion criterion, patients should not have musculoskeletal changes and important visual. The research respected and followed the criteria of ethics in research with human beings recommended in Resolution 466/12 of the National Council of Ethics in Research and, all participants of the research signed the informed consent form (TCLE). 


\section{Otolaryngology Open Access Journal}

The protocol adapted from Cawthorne and Cooksey [13] has ten phases, gradually increasing the complexity of the exercises. The patients are instructed to perform exercises at home daily, three times a day, every day. The exercises consist of movement and fixation of the eyes, head, body and balance activities. In the present study, the exercises were performed concurrently with the use of the X-Box 360 video game, which is a device based on virtual reality, allowing the interaction of individuals in real time. The activities with the X-Box 360 were developed individually and in pairs. The Dizzeness Handicap Inventory-DHI / Brazilian questionnaire was applied before and after the use of the X-Box 360 video game. This instrument was translated from English into Portuguese and culturally adapted, it has 25 objective questions, divided into subscales corresponding to the following aspects: emotional (7), physical (9) and functional (9) [19]. DHI questions were read aloud and marked by the researcher. Each of the participants responded orally to the questions, which had three possible answers, but only one alternative could be chosen. Participants were asked to answer "no" (absence of symptoms), "yes" (presence of symptoms), "sometimes" (occasional presence of symptoms). Each answer had a specific score, "no" 0 points, "yes" 4 points and "sometimes" 2 points. For analysis it is considered that the score of 0 point (minimum value) indicates that dizziness does not cause impairment in quality of life and the closer to 100 points (maximum value) the greater the interference of dizziness in the individual's daily activities and, consequently, worse quality of life.

In each session, before the activities with the X-Box 360 , exercises for relaxation of the shoulder girdle were performed. The exercises consisted of movements of relaxation and rotation of the shoulder, flexion, extension, rotation and lateralization of the head, in order to relax and prepare the body for later activities. The individuals were instructed to go to the sessions wearing light clothes, in order to facilitate movements and / or enable comfort, and to perform barefoot activities, in order to allow greater body perception and proprioceptive stimuli.

In the X-Box 360, the CDs Kinect Adventures and Kinectsport were used, the first with games, such as space bubble, rapids, top of reflexes, ricochets and leaks, where individuals should burst bubbles, deflect, squat and jump obstacles, knock down blocks and close leaks, respectively. The second contains the following sports: volleyball, bowling, table tennis, football and athletics. Both allowed the work of motor and cognitive skills, such as proprioception, agility, coordination, spatial and temporal notion, memory, understanding and quick decision making for what was requested.

The games selected for each session were in accordance with the functional capacity of each individual. Initially Kinect Adventures games were selected and after a few sessions Kinectsport games. In some of these games, individuals were on a balance disc or on two "semicircles", moving according to the objectives of the game. Information was collected throughout the research. For that purpose, the evolution form was used, containing the following data: identification, age, professional occupation and date, in addition to the weekly record of clinical conditions presented by each patient, which allowed the presence of dizziness, vertigo, headache and tinnitus to be monitore during the activities developed in daily life and in order to check if the Cawthorne and Cooksey [13] protocol exercises were performed daily. The researcher individually questioned and noted the clinical evolution of each patient during each session.

The DHI data before and after the intervention were compared and analyzed quantitatively, generating graphs and tables for better visualization, as well as the description of the results, which were treated by descriptive statistics using simple frequencies.

\section{Results}

The sample consisted of five individuals, four female $(80 \%)$ and one male (20\%) (Graph 1), with an age range of 58 to 61 years and an average age of 59.6 years.

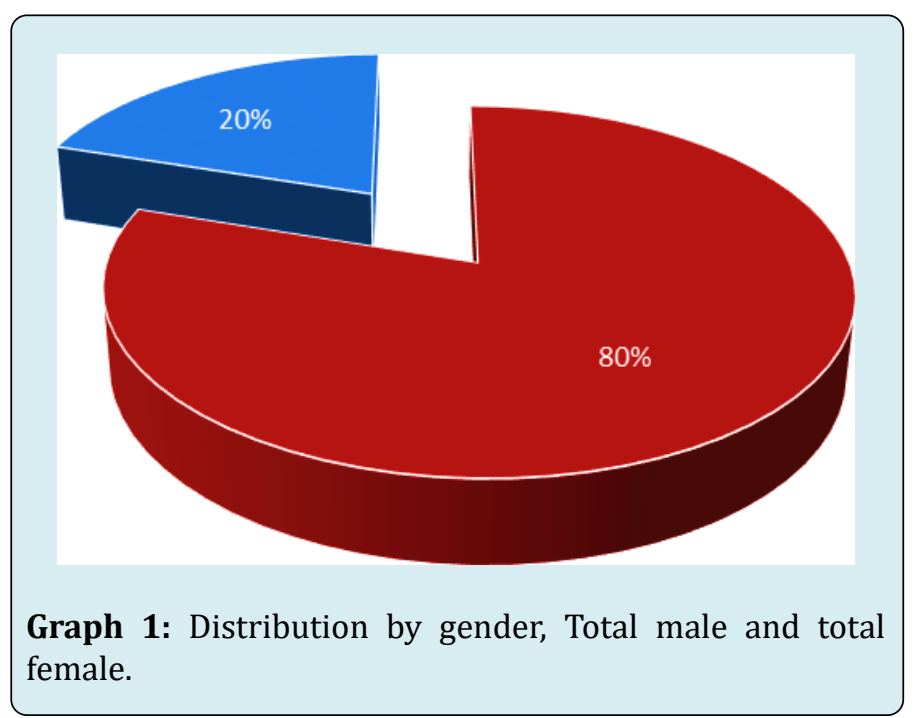

The Dizziness Handicap Inventory or DHI / Brasileiro [19] was applied before and after RV. Thus, before the intervention, it was found that $100 \%$ of the individuals had impairment in the physical domain, $80 \%$ in the functional domain and $80 \%$ in the emotional domain. After intervention, it was found that $40 \%$ had impairment in the physical domain, $100 \%$ functional and $60 \%$ in the emotional domain (Table 1). 


\section{Otolaryngology Open Access Journal}

\begin{tabular}{|c|c|c|}
\hline \multirow{2}{*}{ Domain } & \multicolumn{2}{|c|}{ No. of subjects with presence of alteration } \\
\cline { 2 - 3 } & Before & After \\
\hline \multirow{2}{*}{ Physical } & $\mathrm{N}=5$ & $\mathrm{~N}=2$ \\
& $100 \%$ & $40 \%$ \\
\hline \multirow{2}{*}{ Functional } & $\mathrm{N}=4$ & $\mathrm{~N}=5$ \\
& $80 \%$ & $100 \%$ \\
\hline \multirow{2}{*}{ Emotional } & $\mathrm{N}=4$ & $\mathrm{~N}=3$ \\
& $80 \%$ & $60 \%$ \\
\hline
\end{tabular}

Table 1: Impact of dizziness on quality of life according to the domain before and after intervention.

For a better understanding of the data obtained, individual analysis was also performed before and after speech therapy intervention according to the studied domains (Tables 2 and 3). From the DHI score and subsequent comparison of the scores, the presence or absence of impairment of the physical, functional and emotional domain was identified, with improvement in the interference of dizziness.

\begin{tabular}{|c|c|c|c|c|}
\hline Subject & D. Physical & D. Functional & D. Emotional & Total \\
\hline S1 & 10 & 0 & 2 & 12 \\
\hline S2 & 8 & 8 & 4 & 20 \\
\hline S3 & 12 & 4 & 0 & 16 \\
\hline S4 & 10 & 14 & 18 & 42 \\
\hline S5 & 8 & 6 & 12 & 26 \\
\hline
\end{tabular}

Caption D: domain.

Table 2: Individual DHI before speech therapy intervention according to the domains.

\begin{tabular}{|c|c|c|c|c|}
\hline Subject & D. Physical & D. Functional & D. Emotional & Total \\
\hline S1 & 10 & 8 & 10 & 28 \\
\hline S2 & 2 & 6 & 4 & 12 \\
\hline S3 & 6 & 2 & 0 & 8 \\
\hline S4 & 2 & 10 & 18 & 30 \\
\hline S5 & 4 & 4 & 0 & 8 \\
\hline
\end{tabular}

Caption D: domain.

Table 3: Individual DHI after speech therapy intervention according to the domains.

The impact of dizziness on quality of life was also verified according to age (Table 4), in which the impact is represented

by the DHI score.

\begin{tabular}{|c|c|c|c|c|}
\hline Subject & Sex & Age & Before intervention & After intervention \\
\hline S1 & F & 61 & 12 & 28 \\
\hline S2 & F & 61 & 20 & 12 \\
\hline S3 & F & 58 & 16 & 8 \\
\hline S4 & M & 60 & 42 & 30 \\
\hline S5 & F & 58 & 26 & 8 \\
\hline
\end{tabular}

Caption: F: feminine; M: male.

Table 4: Impact of dizziness according to sex and age. 


\section{Otolaryngology Open Access Journal}

For analyze the improvement in the individuals' quality of life, the total DHI score was verified before and after speech therapy intervention (Table 5).

\begin{tabular}{|c|c|c|}
\hline Subject & Before the Intervention & After Intervention \\
\hline S.1 & 12 & 28 \\
\hline S. & 20 & 12 \\
\hline S.3 & 16 & 30 \\
\hline S.4 & 42 & 8 \\
\hline S.5 & 26 & 8 \\
\hline
\end{tabular}

Table 5: DHI before and after speech therapy intervention.

Dizziness, vertigo, headache, tinnitus and emesis were the clinical symptoms commonly reported by patients and recorded in the progress chart. Most participants said they performed the Cawthorne and Cooksey [13] protocol exercises once a day, two or three times a week.

\section{Discussion}

The aging process causes several health changes in the elderly population. Individuals with dizziness have several clinical characteristics, which cause a compromise in the quality of life [21], resulting in social and psychological changes that compromise the practice of daily activities [19]. The clinical symptoms frequently described in the evolution chart were compatible with those found in the literature [15]. According to the study, such symptoms are commonly found in individuals with labyrinthine alteration, causing a reduction in daily, work and social activities.

It was found that the Cawthorne and Cooksey protocol [13] exercises were performed once or twice a week, the same should be performed two to three times a day throughout the week at home. Note the failure to carry out the requested guidelines and the hypothesis that the final result would be much more favorable, such as a greater decrease in symptoms if the exercises were performed according to recommendations. When questioned, the research subjects reported lack of time to perform the exercises, due to domestic demands and / or care for family members, especially grandchildren. The success of the treatment or the recovery closer to normality will depend on the collaboration and commitment of the individual [16]. Dysfunctions in body balance resulting from the impairment of the vestibular system can cause damage to the physical, emotional, functional factor and produce social and physical dependence [22].

After the intervention with the X-Box 360 video game, it was found that $40 \%$ of the participants had impairment in the physical domain, with a $60 \%$ reduction in the impact of dizziness, $60 \%$ impairment in the emotional domain, showing a $20 \%$ reduction in the impact of dizziness in quality of life and $100 \%$ in the functional domain, revealing a $20 \%$ increase in the impact of dizziness. Thus, there was a reduction in the impact of dizziness in the physical and emotional domain and an increase in the functional domain. Despite the increase in the latter, there is an improvement in quality of life, presented by the final DHI score in most of the individuals surveyed. It is worth mentioning that at the beginning of the research, one of the individuals (S1) did not present any change in the functional domain, and, during rehabilitation, he reported complaints, as he went into crisis during the intervention period, not performing the exercises indicated at home, as had fear of worsening, which caused an increase in the score of the domain surveyed. It is known that individuals with vestibulopathy must have a healthy lifestyle, such as adequate food and weight, greater water intake, physical activity, as well as eliminating or reducing smoking, avoiding the use of drugs without medical prescription, avoiding drinks alcohol, fatigue, stress and anxiety. Such factors may favor the improvement of the symptoms of dizziness and the associated clinical characteristics, thus allowing a more favorable prognosis [23].

As previously mentioned, in addition to analyzing in general the impact of dizziness according to the domains, there was also an individual analysis of each participant after intervention. Thus, $\mathrm{S} 1$ worsened in the symptoms of dizziness and consequently worsened in the physical, functional and emotional domains. However, S2, S3, S4 and S5, improved in the physical, functional and emotional domains after speech therapy intervention.

The physical domain is often the aspect with the greatest impairment in individuals affected by dizziness, followed by the functional and emotional domain $[19,21]$. The physical domain, which was the most altered before the intervention, revealed in DHI, answers with a higher score when moving the head quickly, looking up, walking on the sidewalk, tilting the body and walking in the supermarket. In the functional domain, difficulties in lying down and / or getting out of bed, concentration, restriction on leisure and work trips, 


\section{Otolaryngology Open Access Journal}

worsening in the performance of domestic activities such as washing dishes and sweeping were the most scored responses in DHI. In the emotional domain, the participants showed responses to DHI, with a high score. When asked, they felt depressed, incapacitated, afraid of being alone at home, frustrated, ashamed of associating them with drunk and / or drugged individuals, these findings were similar to those found in the literature [19].

In the present study, it appears that $80 \%$ of the individuals are female and $20 \%$ male, corroborating the literature in which women have greater complaints and incidence of dizziness $[21,24]$. The symptoms are attributed to metabolic and hormonal changes commonly found in women, reducing the performance of the vestibular system $[25,26]$. It is worth mentioning that women have greater health care and, consequently, greater demand for health services than men.

Dizziness in adults and the elderly is a matter of great relevance for professionals who develop activities in this age group. The study shows that $40 \%$ of individuals were 58 years old, $40 \%$ were 61 years old and $20 \%$ were 60 years old. These findings corroborate the literature, which points out that dizziness is more often found in elderly individuals than in young people $[2,19]$. In addition, the functional sequelae in elderly individuals can be very different than in young individuals, due to other existing comorbidities.

In presbyvertigo, there is a degeneration of the systems involved with balance and thus there is a higher incidence of elderly people in vestibular rehabilitation8. With age, the vestibular system degenerates, causing dizziness, associated with the appearance of body imbalance, falls and hearing loss [2]. In this study, individuals with younger ages, 58 and 60 years old, showed an improvement in quality of life after speech therapy intervention, one older person (61 years old) improved, but the other one presented a worse quality of life. Thus, it was found that age did not influence the achievement of better results in quality of life, since one of the older participants also obtained a satisfactory result, corroborating the literature [27].

After speech therapy intervention with the use of the $\mathrm{X}$-Box 360 video game, we can see that of the five individuals surveyed, S1 did not show any improvement in dizziness (20\%), however four individuals (80\%) showed a decrease in the complaint. Daily activities, such as walking the aisles of the supermarket, sweeping the house, storing dishes, not feeling depressed and / or frustrated, quick movements of the head, changing position in bed, directing the look up, among others, do not most worsened or triggered dizziness after intervention.
Our findings were in accordance with a study [28] in which the use of the X-Box 360 associated with conventional exercises enabled a new approach in restoring body balance, as well as improving the performance of daily activities, bringing benefits in quality of life, allowing greater interactivity and fun with group participation. Virtual reality collaborates to considerably maximize the quality of life of individuals who use this tool. It allows immersion in an artificial environment where the perception of that environment is altered by sensory stimuli that can cause a vestibulo-ocular conflict, modifying the gain of this same reflex [29].

Another relevant factor is the individuals' self-perception regarding the use of the X-Box 360 video game to reduce dizziness. A survey of seven members of a Clinical School of Speech Therapy in Salvador-Ba, verified the perception of individuals with the use of the X-Box 360 video game. Some individuals considered the video game to be an alternative tool for vestibular rehabilitation and others reported that the it could even replace conventional protocols [28]. The use of recreational rehabilitation with the X-Box 360 (X Box therapy), collaborates in the treatment of the elderly, since they have the perception of a relaxed environment, favoring adherence to therapy, providing individuals with affirmative actions about cognition, autonomy, balance and functionality5. In addition, although the use of virtual games promotes benefits for the elderly, they show concern in their use, because without the assistance of a specialized professional, their use may cause worsening of symptoms [30]. In another study, individuals reported greater social interaction, confidence, pleasure, willingness to carry out activities and mainly an improvement in quality of life [31]. Thus, virtual reality, through games, can provide interaction to the patient, through the very balance relationships established by the sensation of experiencing a new reality $[32,33]$.

\section{Study Limitations}

Despite the benefits found in the present research, there is a shortage of national studies that ratify the effectiveness of the X-Box 360 video game in vestibular rehabilitation, as well as the small number of individuals surveyed for greater relevance of the results obtained.

\section{Conclusion}

The present study demonstrated that there were benefits in the quality of life of most participants, such as reduced dizziness, decreased impairment in the physical, functional and emotional aspects, providing improvement in the performance of daily activities, verifying the effectiveness of using the video game X- Box 360, in the vestibular 


\section{Otolaryngology Open Access Journal}

rehabilitation of individuals affected by dizziness.

\section{References}

1. Carvallo Filho ET (2002) Physiology of aging. In: Papaléo Neto M (Eds.), Gerontologia, São Paulo: Atheneu pp: 6070 .

2. Santana GG, Doná F, Ganança MM, Kasse CA (2011) Vestibulopathy in the elderly. Health Colet 8(48): 52-56.

3. Bittar RSM, Oiticica J, Bottino MA, Ganança FF, Dimitrov $R$ (2013) Population epidemiological study on the prevalence of dizziness in the city of São Paulo. Braz J Otorhinolaryngol 79(8): 688-698.

4. Mantello EB, Moroguti JC, Júnior ALR, Ferrioloi E (2008) Effect of vestibular rehabilitation on the quality of life of the elderly with labyrinth disease. Rev Bra de Otorrinolaringol 74(2): 172-180.

5. Borges APM, Mendes GC (2015) Cognitive and balance assessment in institutionalized elderly people after X Box therapy intervention. Cad da Esc de Saúde 1(3): 93104.

6. Caovilla HH, Ganança MM, Munhoz MS, Silva ML (1999) Clinical balance. São Paulo: Editora Atheneu.

7. Simocelli LR, Bittar MS, Bottino MA, Bento RF (2003) Diagnostic profile of the elderly with body imbalance: preliminary results. Rev Bras de Otorrinolaringol 69(6): 772-777.

8. Doná F, Araújo JPF, Maia DAR, Alves AM, Kasse CA (2011) Electronic games in the rehabilitation of body balance in the elderly with vestibular disease: clinical case. Rev Uni Vale Rio Verde. 12(1): 693-702.

9. Zanoni A, Ganança F (2010) Virtual reality in vestibular syndromes/Virtual reality in vestibular syndromes. Rev Bras Med 67(1).

10. American Speech-Language-Hearing Association (ASHA). Dizziness and vertigo.

11. Takano NA, Cavalli SS, Ganança MM, Caovilla HS, Oliveira MA, et al. (2010) Quality of life of elderly people with dizziness. Braz J Otorhinolaryngol 76(6): 769-775.

12. Pereira PC, Oliveira LHS, Souza VV, Silva AS (2013) Effectiveness of vestibular rehabilitation in elderly with dizziness. Rev Uni Vale Rio Verde 11(2): 371-378.

13. Poster session (2008) 16 Brazilian Congress of Speech Therapy; Campos do Jordão (SP): University of the State of Bahia-UNEB / UATI.
14. Canto MGM, Fonseca AMP (1997) Labyrinth rehabilitation: an alternative way to treat vestibular disorders. Monograph presented to UFBA as a final requirement of the $1^{\text {st }}$ Specialization Course in Speech Therapy - Audiology Concentration Area]. Salvador (BA): Institute of Health Sciences, Federal University of Bahia.

15. Kesller N, Ganança MM, Ganança CF, Ganança FF, Lopes SC, et al. (2012) Balance Rehabilitation Unit (BRU ${ }^{\mathrm{TM}}$ ) posturography in relapsing-remitting multiple sclerosis. Arq Neuropsiquiatr 69(3): 485-490.

16. Mirallas NDR, Conti MHS, Vitta, AD, Saes SO (2011) Vestibular assessment and rehabilitation in the elderly. Rev Bras Geriatr Gerontol 14(4): 687-698.

17. Proceedings (2012) XX Brazilian Congress of Speech Therapy Brasília (DF): State University of Bahia-UNEB.

18. Grande AAB, Galvão FRO, Gondim LCA (2012) Virtual rehabilitation through videogame case report in the treatment of a patient with high lesion of the median and ulnar nerves. Acta Fisiátr 18(3): 157-162.

19. Full Papers (2012) XI SB Games; 2-4 Nov 2012; Brasília (DF): Lutheran University Center of Palmas.

20. Castro AS, Gazzola JM, Natiour J, Ganança FF (2007) Brazilian version of the Dizziness Handicap Inventory. Rev Pró-Fono 19(1): 97-104.

21. Rivera IDS, Zeigelboim BS, Jurkiewicz AL (2003) Vestibular rehabilitation in pregnant women with benign paroxysmal positional vertigo: study of two Cases. Acta ORL 21(2).

22. Sherer S, Lisboa HRK, Pasqualotti A (2012) Dizziness in the elderly: otoneurological diagnosis and interference with quality of life. Rev Soc Bras Fonoaudiol 17(2): 4250.

23. Santos EM, Gazzola JM, Ganança CF, Caovilla HH, Ganança FF (2010) Impact of dizziness on the quality of life of the elderly with chronic vestibulopathy. Rev Pró Fono 22(4): 427-432.

24. Ganança MM, Caovilla HH, Ganança FF, Doná F, Branco F, et al. (2008) How to diagnose and treat vertigo. Rev Bras Med 65(12): 6-14.

25. Morozetti PG, Ganança CF, Chiari BM (2011) Comparison of different vestibular rehabilitation protocols in patients with peripheral vestibular disorders. J Soc Bras Fonoaudiol 23(1): 44-50.

26. Bittar RSM, Bottino MA, Zerati FE, Morais CLO, Cunha AS (2003) Prevalência das alterações metabólicas em 


\section{Otolaryngology Open Access Journal}

pacientes portadores de queixas vestibulares. Rev Bras Otorrinolaringol 69(1): 64-68.

27. Tavares FS, Santos MFC, Knobel KAB (2008) Vestibular rehabilitation in a university hospital. Rev Bras Otorrinolaringol 74(2): 241-247.

28. Ricci NA, Aratani MC, Doná F, Macedo C, Caovilla HH, et al. (2010) Systematic review on the effects of vestibular rehabilitation in middle-aged and elderly adults. Rev Bras Fisioter 14(5): 361-367.

29. Anais (2014) XXVIII Encontro International de Audiologia, Florianópolis (SC): Universidade do Estado da Bahia.
30. Yamamoto MEI, Ganança CF (2012) Posturography with virtual reality stimuli in different vestibular disorders. Rev Soc Bras Fonoaudiol 17(1): 54-60.

31. Bushatsky A, Lebrão ML (2009) Body balance, aging and vestibular rehabilitation: implications for public health. Saúde Co-let 30(6): 102-103.

32. Anais (2015) 30 Encontro Internacional de Audiologia, Bauru (SP): Universidade do Estado da Bahia.

33. Nascimento JAA, Rocha MFMR, Silva JK, Santos SAS (2019) Use of Virtual Games as a Tool in Rehabilitation Physiotherapeutic Vestibular the Elderly. J Bras Tele 6(1): 79-85. 